\title{
Compressibilité d'un sédiment de dragage traité au ciment à forts rapports $\mathrm{E} / \mathrm{C}$
}

\author{
Boubaker REKIK \\ Ecole Supérieure des Ingénieurs de Poitiers \\ 40 avenue du Recteur Pineau, 86000 Poitiers, France \\ Boubaker.rekik@univ-poitiers.fr
}

\section{Résumé :}

Dans cet article, nous discutons du comportement en compressibilité d'un sédiment avant et après traitement. Des essais ont été réalisés sur deux sédiments de teneurs en matières organiques MO différentes (7 et 2\%) avant et après traitement avec 2, 5 et $10 \%$ de ciment après 7 et 28 jours de cure. Les résultats montrent une augmentation de la pression de préconsolidation, une diminution de la compressibilité, une accélération de la phase de consolidation et une réduction de la compression secondaire. Les propriétés après traitement sont d'autant plus intéressantes que la teneur en MO est faible.

\section{Mots clés:}

Sédiment - Ciment - Matières organiques MO - Compressibilité.

\begin{abstract}
:
In this paper, we discuss the effects of cement content and organic matter content on the compressibility behaviour of treated and non treated sediments. The sediments of a water content of $120 \%$ were treated with 2,5 and $10 \%$ of cement and tests performed after 7 and 28 days of curing. Results confirm an increase in the preconsolidation pressure, a decrease in the compressibility rate, an accelerating of the consolidation process and a reduction in the secondary compression. Moreover, more interesting properties are obtained if the OM content is reduced from 7 to $2 \%$ prior to cement treatment.
\end{abstract}

\section{Keywords:}

Sediment - Cement - Organic matter OM - Compressibility.

\section{Introduction}

La gestion des sédiments de dragage est l'une des difficultés que rencontrent les autorités et les gestionnaires des infrastructures portuaires et fluviales. La 
valorisation de ces matériaux dans des applications géotechniques telles que le remblaiement, le remplissage des tranchées ou les couches routières constituent une alternative possible au rejet en mer et au stockage. Cependant, ces matériaux présentent des propriétés mécaniques et géotechniques médiocres qui empêchent de telles valorisations. Le traitement par des liants hydrauliques est utilisé depuis plusieurs décennies pour améliorer les propriétés mécaniques et géotechniques des sols fins (CHEW et al., 2004). Depuis quelques années, dues au durcissement des règlements en matière de gestion des sédiments de dragage, la solidification/stabilisation de ces matériaux par des liants hydrauliques est encouragée. Les résultats montrent une augmentation de la résistance mécanique et une amélioration des propriétés géotechniques après traitement. En effet, lorsque les sols et les sédiments fins sont traités, le comportement géotechnique passe de celui d'un sol fin vers celui d'un sol plus grossier. Ce changement se manifeste principalement par une accélération de la phase de consolidation primaire (réduction du temps de fin de consolidation primaire $t_{100}$ ) et une réduction de la compression secondaire. En plus, la résistance mécanique augmente lorsque le rapport eau/ciment diminue (BOUTOUIL \& LEVACHER, 2005). Cela signifie que de meilleures propriétés mécaniques sont obtenues si une déshydratation préalable est effectuée.

Outre la teneur en eau élevée, ces matériaux contiennent généralement entre 5 et $10 \%$ de MO. Ces substances interfèrent avec les argiles pour former des agrégations très poreuses augmentant la compressibilité (STEVENSON, 1994). De plus, l'augmentation de la teneur en OM augmente la plasticité et la compression secondaire et diminue la perméabilité (RASHID \& BROWN, 1975 ; MITCHELL, 1993). L'effet de la teneur en MO devient prédominante sur le comportement géotechnique lorsqu'elle dépasse 4-5\% (KELLER, 1982); d'autres études montrent que ce paramètre influence les propriétés géotechniques même pour des teneurs plus faibles que 3\% (BOUTH \& DAHL, 1985). Lorsqu'un traitement au ciment est envisagé, la MO peut perturber les réactions du liant en retardant le processus d'hydratation ou en l'inhibant (Tremblay et al., 2002). Ces perturbations se manifestent par une réduction de l'efficacité du traitement et de la résistance mécanique du matériau traité.

Dans ce papier, nous discutons l'effet de la teneur en MO sur le comportement géotechnique des sédiments de dragage. Pour cela, des essais œdométriques ont été réalisés sur deux sédiments de même minéralogie et contenant 2 ou $7 \%$ de MO avant et après traitement avec 2, 5 et $10 \%$ de ciment. Les essais sur les sédiments traités ont été effectués après une cure de 7 et 28 jours. Des analyses MEB et de 
diffraction aux rayons $\mathrm{X}$ ont été faites afin d'apprécier l'effet de la microstructure sur les paramètres caractéristiques de la compressibilité de ces matériaux.

\section{Méthode et matériaux}

Cette étude a été réalisée sur un sédiment marin prélevé du port de Ouistreham (France). Ses caractéristiques déterminées selon les normes AFNOR sont résumées dans le Tableau 1. Les teneurs en eau et en $\mathrm{MO}$ obtenues par séchage à $50^{\circ} \mathrm{C}$ et par calcination à $450^{\circ} \mathrm{C}$ respectivement sont de 120 et de $7 \%$. Les limites de liquidité $w_{L}$ et de plasticité $w_{P}$ sont de 104 et de $38 \%$ et l'indice de plasticité $I_{P}$ de $66 \%$. Cette forte plasticité est due à la présence d'une forte proportion d'argiles (i.e., 25\%) associée à 7\% de MO. La diffraction aux rayons $\mathrm{X}$ a montré que le quartz et la calcite sont les principaux minéraux dans ce sédiment. Les espèces argileuses détectées sont la smectite, l'illite, les micas et la kaolinite.

Tableau 1. Propriétés du sédiment brut du port de Ouistr
\begin{tabular}{|c|c|}
\hline Propriétés & Valeur \\
\hline Granulométrie & \\
Sable $(>63 \mu \mathrm{m})(\%)$ & 25 \\
Limon $(2-63 \mu \mathrm{m})(\%)$ & 50 \\
Argile $(<2 \mu \mathrm{m})(\%)$ & 25 \\
\hline Teneur en eau (\%) & 120 \\
Teneur en MO (\%) & 7 \\
Valeur au bleu (g/100g) & 3,4 \\
Limite de liquidité (\%) & 104 \\
Limite de plasticité (\%) & 38 \\
Indice de plasticité (\%) & 66 \\
\hline
\end{tabular}

Afin de mesurer l'effet de la teneur en MO sur la compressibilité, un deuxième sédiment avec une teneur en MO de $2 \%$ a été préparé par oxydation du sédiment brut $(7 \%$ de $\mathrm{MO})$ à l'eau oxygénée $\left(\mathrm{H}_{2} \mathrm{O}_{2}\right)$. Celui-ci a été lavé à l'eau de mer afin de restituer les conditions de salinité préexistantes avant traitement à l'eau oxygénée. Finalement, sa teneur en eau a été réduite à $120 \%$ par séchage à l'air libre. Les sédiments avec 2 et $7 \%$ de MO ont été traités à la teneur en eau initiale (i.e., 120\%) avec 2, 5 ou 10\% de ciment CEM II/B-M (LL-S) 32,5 (dosages exprimés par rapport à la masse humide du sédiment). Les mélanges cimentsédiments ont été conservés dans des boites hermétiques à $20^{\circ} \mathrm{C}$ et une humidité relative de $80 \%$ pendant 7 et 28 jours.

Les essais de compression à l'œdomètre ont été effectués selon la norme AFNOR XP P 94-090-1. Ces essais ont été réalisés sur le sédiment non traité sur 
des échantillons de teneur en eau de $120 \%$. Sur les différents mélanges cimentsédiment, les mêmes essais ont été effectués après 7 et 28 jours de cure. De plus, des analyses au MEB et par diffraction aux rayons $\mathrm{X}$ ont été faites dans le but de comprendre l'effet de la microstructure des mélanges sur les paramètres de consolidation du sédiment.

\section{Résultats}

\subsection{Compressibilité du sédiment non traité}

Les courbes de compression concernant les sédiments non traités données par les Figures 1 sont linéaires sans pression de préconsolidation $\sigma_{p c}^{\prime}$. Ce type de courbes est caractéristique pour ces matériaux du fait de leur remaniement permanent qui ne permet pas le développement d'une structure réelle. Les résultats montrent que sous le même incrément de contrainte, l'indice des vides du sédiment contenant $7 \%$ de $\mathrm{MO}$ est supérieur à celui du sédiment avec $2 \%$ de $\mathrm{MO}$. Ceci est dû aux propriétés hydrophiles des substances organiques qui se comportent comme une éponge, retiennent l'eau et empêchent le drainage par augmentation des pressions capillaires (MITCHELL, 1993).

Le sédiment dragué, contenant $7 \%$ de $\mathrm{MO}$ a un indice de compression $C_{c}$ de 0,74 . La réduction de la teneur en $\mathrm{MO}$ à $2 \%$ permet de réduire cet indice à 0,55 . De plus, elle permet d'augmenter le coefficient de consolidation primaire $C_{v}$ de 0,3 à $0,8 \mathrm{~m}^{2} /$ an et de réduire son coefficient de compression secondaire $C_{\alpha}$ légèrement de 0,03 à 0,02 . Ces résultats signifient qu'une réduction de la teneur en MO réduit la compressibilité du sédiment accélère le processus de consolidation primaire. Cependant, il semble qu'avec de telles teneurs, la MO n'a pas d'effets significatifs sur la compression secondaire. Ainsi, excepté ces constatations sur la compression secondaire, les résultats se concordent parfaitement avec ceux rapportés par Rashid \& Brown, (1975). De plus, le rapport $C_{\alpha} / C_{c}$ est intrinsèque au sol (HOLTZ et al., 1996). Ce rapport est inversement proportionnel à la taille moyenne des grains. Pour ce sédiment, $C_{d} / C_{c}$ est de 0,036 et il n'a pas changé après la réduction de la teneur en MO. Cette valeur est moyenne par rapport à celles données dans la littérature sur les sols argileux et limoneux organiques.

Les Figures 1 illustrent les courbes de compression du sédiment contenant 2 et $7 \%$ de $\mathrm{MO}$ avant et après traitement avec respectivement 2,5 et $10 \%$ de ciment. Elles montrent un changement progressif de l'allure des courbes après traitement lorsque le dosage en ciment augmente. Ainsi, l'augmentation du dosage en ciment diminue l'indice des vides et la compressibilité du sédiment alors que $\sigma_{p c}^{\prime}$ augmente. Pour mieux cerner l'influence du traitement, les paramètres caractéristiques de la compressibilité ont été déterminés. 

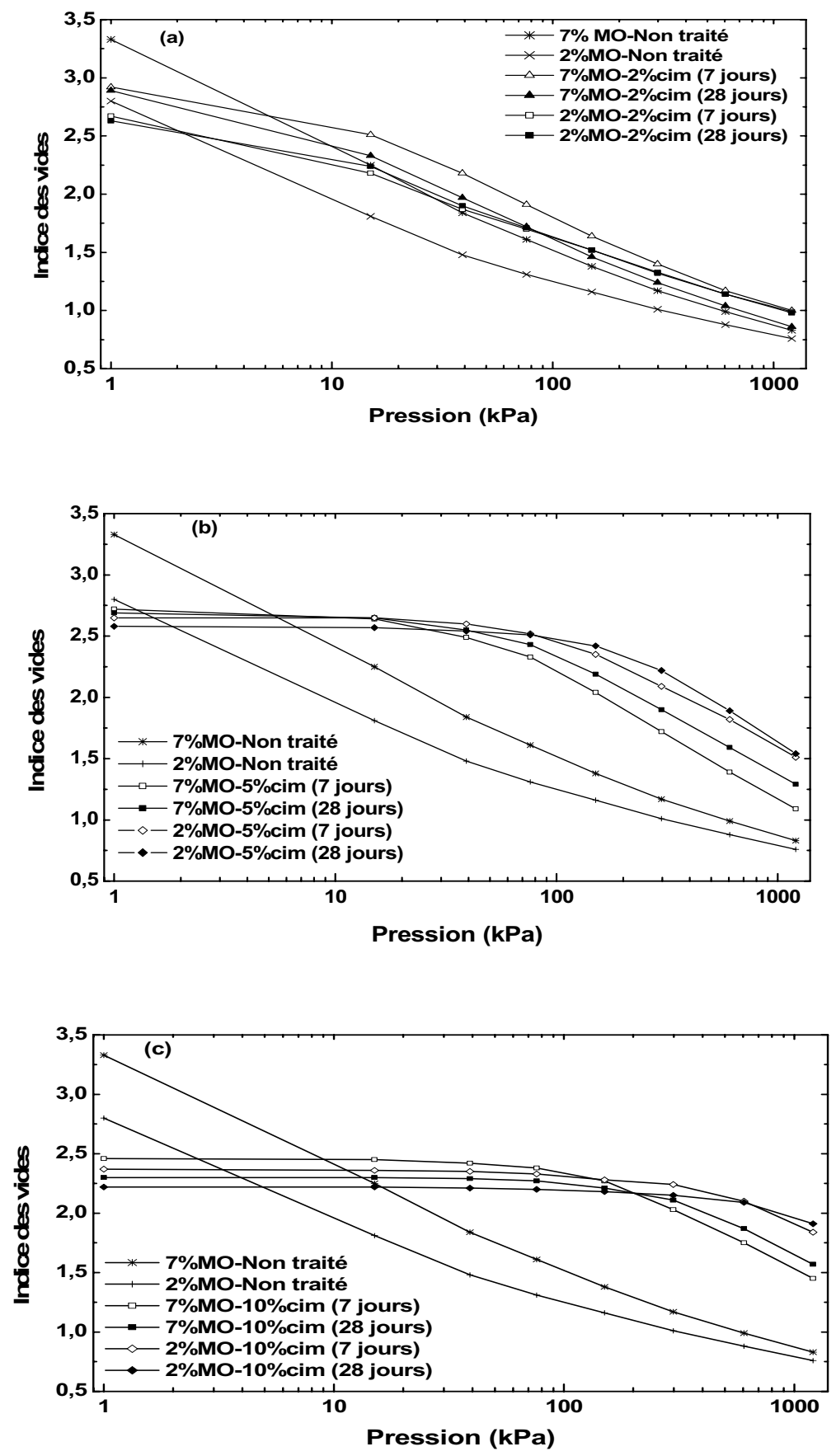

Figure 1. Courbes de compression du sédiment contenant 2 et $7 \%$ de MO traité avec: (a) 2\% de ciment, (b) 5\% de ciment, (c) 10\% de ciment.

L'augmentation du dosage en ciment réduit les indices des vides initiaux suite à une réduction de la teneur en eau et un comblement progressif des pores par dépôt d'hydrates. Cet effet commence dès les premières heures et se poursuit avec le 
temps de cure. En effet, on peut constater des courbes de compressibilité que pour le même dosage en ciment que cet indice diminue pour atteindre sa plus faible valeur après 28 jours (REKIK, 2007). De plus et faisant référence au comportement avant traitement où l'indice des vides augmente avec la teneur en MO, cette tendance s'inverse après traitement. Cela implique une formation d'un volume plus important d'hydrates à faible teneur en MO ce qui a conduit à une meilleure solidification et une réduction plus conséquente de déformabilité du matériau.

Après traitement avec $2 \%$ de ciment, $\sigma_{p c}^{\prime}$ reste très faible malgré un début d'apparition (Figure 1-a). Sa faible valeur dans ce cas implique qu'un dosage minimum en liant est nécessaire à la solidification du sédiment. Ce dosage dépend de la teneur en eau car la résistance des sols et des sédiments traités au ciment diminue lorsque le rapport eau/ciment augmente. Ainsi, ce rapport est de 27 lors d'un traitement avec $2 \%$ de ciment ce qui est très élevé (BOUTOUIL \& LEVACHER, 2005). Au-delà de $2 \%$ de ciment, les Figures $1-b$ et $\mathrm{c}$ et la Figure 2 montrent une augmentation linéaire de $\sigma_{p c}^{\prime}$ avec le dosage en ciment. Cette pression continue d'augmenter avec le temps de cure et atteint sa valeur maximale après 28 jours (REKIK, 2007). Les résultats confirment également que pour un même dosage en ciment, $\sigma_{p c}^{\prime}$ augmente lorsque la teneur en MO diminue. En effet, pour des traitements avec 5 et $10 \%$ de ciment, $\sigma_{p c}^{\prime}$ est respectivement trois ou deux fois plus élevée dans le cas du sédiment contenant $2 \%$ de MO que dans celui avec $7 \%$ de MO.

Les valeurs de $C_{c}$ après traitement sont données dans le Tableau 2. Les résultats montrent une augmentation de $C_{c}$ après traitement. Pour des dosages en ciment inférieurs ou égaux à $10 \%$, les valeurs de $C_{c}$ restent supérieures à 0,4 ce qui implique que ces matériaux restent fortement compressibles. Pour la même teneur en $\mathrm{MO}, C_{c}$ augmente avec le dosage en ciment et atteint environ 1 lorsque le dosage en ciment atteint $5 \%$. Lorsque le dosage en ciment augmente de 5 à $10 \%$, $C_{c}$ reste constant dans le cas du sédiment contenant 7\% de $\mathrm{MO}$ mais diminue sensiblement pour atteindre 0,6 dans le cas du sédiment contenant $2 \%$ de MO.

L'examen de l'effet du traitement sur la consolidation primaire et sur la compression secondaire de ces matériaux confirme un changement de comportement. En effet, pour la même teneur en $\mathrm{MO}, C_{v}$ augmente avec le dosage en ciment (Tableau 3). De plus, pour un traitement avec 2 ou $5 \%$ de ciment, $C_{v}$ augmente lorsque la teneur en MO diminue et ils sont équivalents pour un traitement avec $10 \%$ de ciment. Le traitement au ciment réduit légèrement $C_{\alpha}$ et le rapport $C_{\alpha} / C_{c}$ ce qui implique une agglomération des fines en particules de tailles 
plus importantes. Ce résultat a été confirmé par les mesures de la distribution granulométrique après traitement détaillées par Rekik. (2007).

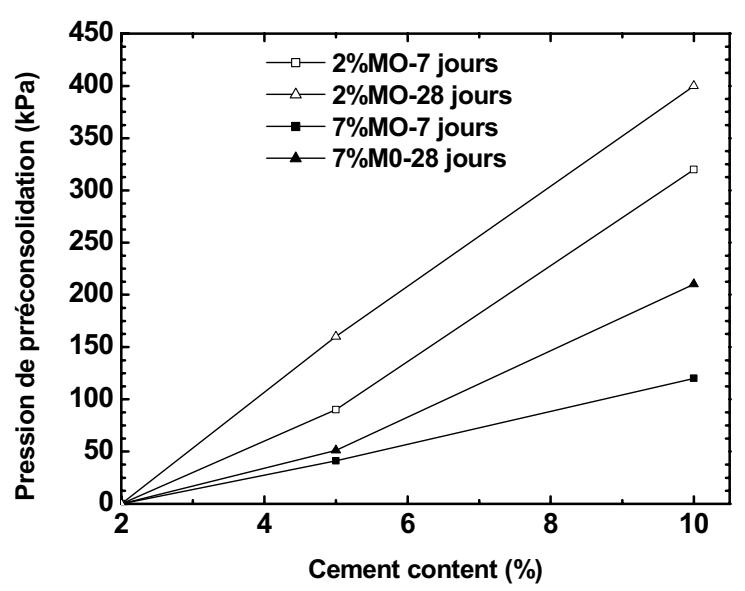

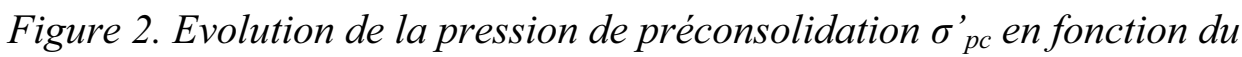
dosage en ciment et de la teneur en MO.

Tableau 2. Indice de compression $C_{c}$ des sédiments contenant 2 et $7 \%$ de $M O$

\begin{tabular}{|c|c|c|c|c|c|c|c|c|}
\hline \multicolumn{1}{|c|}{ avant et après traitement avec 2, 5 et 10\% de ciment. } \\
\hline Age (jours) & & Non traité & \multicolumn{3}{|c|}{7} & \multicolumn{3}{|c|}{28} \\
\hline Ciment (\%) & & & 2 & 5 & 10 & 2 & 5 & 10 \\
\hline \multirow{2}{*}{ MO (\%) } & 2 & 0,55 & 0,59 & 0,96 & 0,59 & 0,62 & 1,12 & 0,62 \\
\cline { 2 - 9 } & 7 & 0,74 & 0,79 & 1,05 & 0,97 & 0,74 & 0,99 & 0,98 \\
\hline
\end{tabular}

Tableau 3. Coefficient de consolidation primaire $C_{v}$ et de compression secondaire du sédiment traité au ciment.

\begin{tabular}{|c|c|c|c|c|c|c|}
\hline MO (\%) & \multicolumn{3}{|c|}{2} & \multicolumn{3}{|c|}{7} \\
\hline Ciment (\%) & 2 & 5 & 10 & 2 & 5 & 10 \\
\hline $\mathrm{C}_{\mathrm{v}}\left(\mathrm{m}^{2} / \mathrm{an}\right)$ & 3,8 & 11,8 & 12,8 & 0,9 & 3,7 & 14,2 \\
\hline $\mathrm{C}_{\alpha}$ & 0,01 & 0,01 & 0,01 & 0,01 & 0,01 & 0,01 \\
\hline $\mathrm{C}_{\alpha} / \mathrm{C}_{\mathrm{c}}$ & 0,010 & 0,005 & 0,007 & 0,022 & 0,009 & 0,005 \\
\hline
\end{tabular}

\subsection{Microstructure}

La microstructure du sédiment a été étudiée par des observations au MEB. Les micrographies $\mathrm{A}$ et $\mathrm{B}$ montrent deux échantillons de sédiments contenant respectivement 7 et $2 \%$ de $\mathrm{MO}$ séchés à $50^{\circ} \mathrm{C}$. Le rétrécissement provoqué lors du séchage empêche d'examiner correctement la microstructure de ces matériaux. Ces micrographies montrent que le sédiment est très fin et contient une forte proportion de grains de taille de l'ordre du micron. Cette fraction semble 
essentiellement de nature argileuse ; elle est associée à divers formes de MO, décomposées ou en cours.
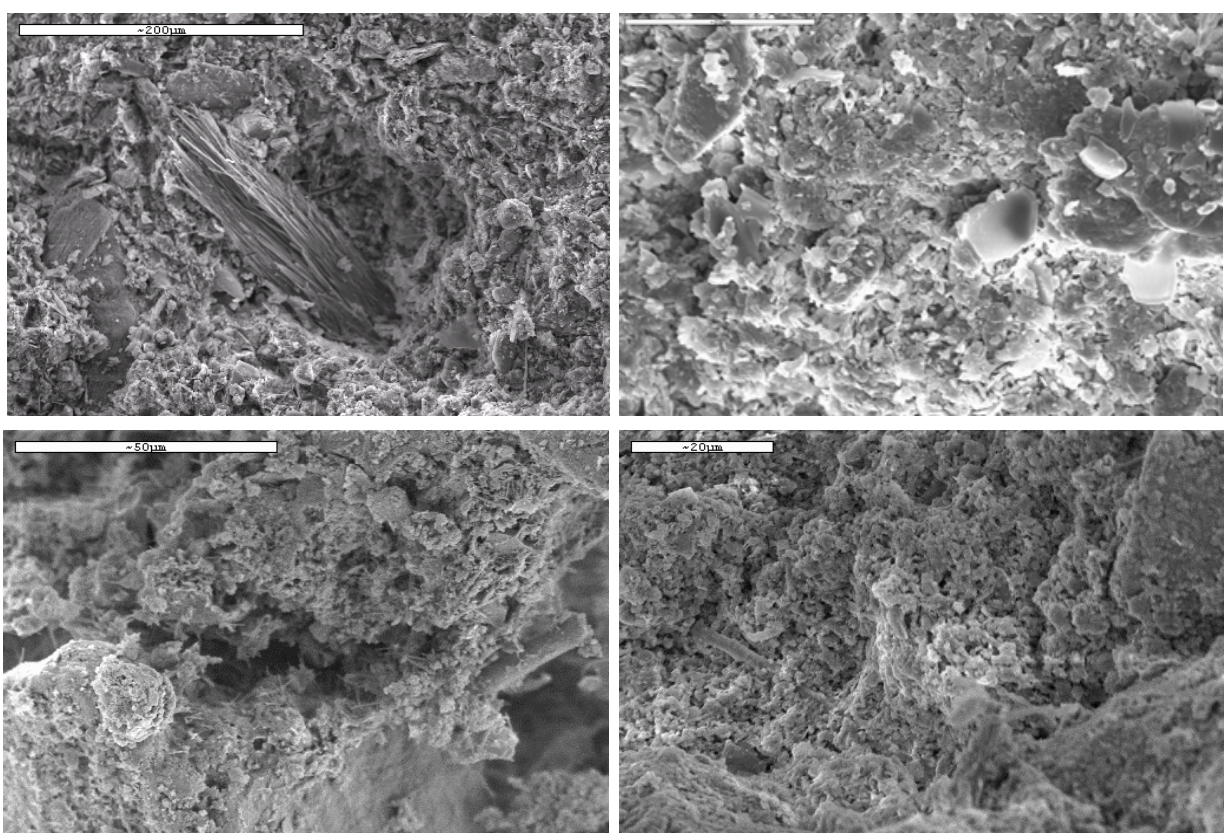

Figure 3. Micrographies des analyses MEB : (A, B) Sédiments non traités contenant respectivement 7 ou $2 \%$ de $M O(C, D)$ Sédiments contenant 7 ou $2 \%$ de MO traits avec $10 \%$ de ciment (28 jours).

Après traitement, les analyses montrent la formation de différents hydrates. Ces composés se forment dès les premiers jours et leur volume augmente avec le dosage en ciment et densifie le sédiment (REKIK, 2007). Les micrographies $\mathrm{C}$ et $\mathrm{D}$ montrent la microstructure des sédiments contenant 7 et $\%$ de MO traités avec $10 \%$ de ciment après 28 jours. Il semble qu'à partir de ces micrographies et des résultats rapportés dans Rekik, (2007) avec une teneur en MO de 7\%, les CSH forment une enveloppe "coque» autour des agglomérations argilo-organiques préexistantes avant traitement. Lorsque la teneur en MO est réduite, ces hydrates se forment autour des grains permettant une meilleure solidification.

Les analyses par DRX sur des les sédiments traités n'ont pas permis d'en savoir davantage sur l'effet de la MO. Les spectres sont quasi similaires à cause des faibles dosages en ciment et de la non cristallisation des hydrates et surtout les $\mathrm{CSH}$ en comparaison avec les phases minérales présentes dans ce sédiment.

\section{Discussion}

Le traitement au ciment permet la solidification du matériau et une amélioration de son comportement géotechnique. En effet, les grains fins s'agglomèrent pendant les premières heures par floculation/agglomération et continue de 
s'agglomérer avec le temps par cimentation (CHEW et al., 2004 ; REKIK, 2007). Cette agglomération modifie le comportement en compressibilité de ces matériaux qui passe progressivement vers celui d'un sol plus grossier (FENG et al., 2001). La cimentation augmente la résistance mécanique et $\sigma_{p c}^{\prime}$.

L'augmentation de $C_{c}$ après traitement est due à la teneur en eau élevée du sédiment. En effet, la cimentation rend le matériau quasi incompressible sous des contraintes inférieures à $\sigma_{p c}^{\prime}$. Près de cette valeur $\sigma_{p c}^{\prime}$, le sédiment traité est plus poreux que celui non traité. Lorsque la contrainte augmente au-delà de $\sigma_{p c}^{\prime}$, elle provoque une destruction partielle de la matrice cimentaire induisant une déformation importante qui se manifeste par des valeurs élevées de $C_{c}$. Quand le dosage en ciment atteint $10 \%$ et surtout dans le cas du sédiment contenant $2 \%$ de $\mathrm{MO}$, le matériau traité devient suffisamment résistant et par conséquent moins compressible. Ceci est confirmé par une réduction de $C_{c}$ de 1 à 0,6 lorsque le dosage en ciment passe de 5 à $10 \%$.

Les propriétés moins performantes obtenues dans le cas du sédiment contenant $7 \%$ de MO confirment l'effet négatif de ces substances sur le traitement au ciment. Cet effet est peut être dû à la nature acide de certaines composés organiques (MITCHELL, 1993).Une partie du ciment introduit sert seulement à réduire cette acidité. Ces composés peuvent réagir avec les ions $\mathrm{Ca}^{2+}$ libérés par la dissolution des silicates ou avec des hydrates formant des carbonates. Cela réduit la quantité de calcium disponible à la formation des CSH.

\section{Conclusion}

La compressibilité du sédiment de dragage a été étudiée avant et après traitement au ciment. L'influence de la teneur en MO et du dosage en ciment a été mise en évidence. Du fait de leur finesse et de leurs teneurs en eau élevées, ces matériaux sont très compressibles. La réduction de la teneur en MO de 7 à $2 \%$ permet de réduire légèrement leur compressibilité et d'accélérer le processus de consolidation primaire. Lorsque le traitement est effectué à une teneur en eau élevée tel est le cas dans cette étude, il réduit leur compressibilité et augmente leur $\sigma_{p c}^{\prime}$. De plus, le traitement au ciment accélère le processus de consolidation primaire et réduit la compression secondaire. Le sédiment traité a un comportement en compressibilité plus proche de celui d'un sol grossier dans le sens où $C_{v}$ augmente et $C_{\alpha}$ diminue.

La teneur en MO est un facteur important lorsqu'un traitement au ciment est envisagé tant de point de vue efficacité du traitement que de point de vue économique. En effet, les résultats de cette étude montrent que les propriétés du sédiment contenant $7 \%$ de MO traités avec $10 \%$ de ciment sont comparables à celles du sédiment contenant $2 \%$ de MO traité avec 5\% de ciment. Cela implique 
qu'une réduction préalable de la teneur en MO permet d'économiser une quantité importante de ciment nécessaire au traitement.

\section{Références}

1 Booth, J.S., Dahl, A.G. (1985). A note on the relationships between organic matter and some geotechnical proprieties of marine sediments, Marine Geotechnology, vol. 6: 281-297.

2 Boutouil, M., Levacher, D. (2005). Effect of high initial water content on cement based treated sludge solidification, Ground improvement, vol. 9, $\mathrm{n}^{\circ} 4$ : 169-174.

3 Chew, S.H., Kamruzzaman, H.M., Lee, F.H. (2004). Physicochemical and engineering behaviour of cement treated clays. Journal of Geotechnical and Geoenvironmental Engineering, vol. 130, n 10: 696-706.

4 Feng, T.W., Lee, J.Y., Lee, Y.J. (2001). Consolidation behaviour of a soft mud treated with small cement content, Engineering Geology, vol. 59: 327-335.

5 Holtz, R.D., Kovacs, W.D., Lafleur, J. (1996). Introduction à la géotechnique. Edition de l'Ecole Polytechnique de Montréal: 808p.

6 Keller, G.H. (1982). Organic matter and geotechnical properties of submarine sediments, Geomarine letters, vol. 2: P. 191-198.

7 Mitchell, J.K. (1993). Fundamentals of soil behaviour, Edition Wiley: 425p.

8 Rashid, M.A., Brown, J.D. (1975). Influence of marine organic compounds on the engineering properties of a remoulded sediment. Engineering Geology, vol. 9: 141-154.

9 Rekik, B. (2007). Propriétés géotechniques des sédiments de dragage traités au ciment. Thèse de doctorat de l'Université de Caen Basse Normandie: 122p.

10 Stevenson, F.G. (1994). Humus chemistry: Genesis, composition, reactions. Edition John Willey and Sons, $2^{\text {nd }}$ Edition: 496p.

11 Tremblay, H., Leroueil, S., Locat J. (2002). Influence of the nature of organic matter on the soil stabilization with cement. Canadian Geotechnical Journal., vol. 39: 535-546.

12 AFNOR, (1997). XP P 94-090-1, Sols: reconnaissance et essais. Essai oedométrique, Partie 1 : Essai de compressibilité sur matériaux fins quasi saturés avec chargement par paliers: 23p. 